\title{
Extinction properties of solutions for a fast diffusion equation with nonlocal source
}

\section{Zhong Bo Fang* and Mei Wang}

\section{"Correspondence:}

fangzb7777@hotmail.com

School of Mathematical Sciences,

Ocean University of China, Songling

Road 238, Qingdao, 266100, P.R.

China

\begin{abstract}
In this paper, we investigate extinction properties of nonnegative nontrivial solutions for an initial boundary value problem of a fast diffusion equation with a nonlocal source in bounded domain. By using the super- and sub-solution and the energy methods, we obtain some sufficient conditions for extinction and non-extinction of the weak solutions and give corresponding decay estimates which depend on the initial data, coefficients, and domains.
\end{abstract}

MSC: 35K65; 35K20; 35B40

Keywords: fast diffusion equation; extinction; non-extinction; decay estimate

\section{Introduction}

We consider the fast diffusion equation with a nonlocal source

$$
u_{t}=\Delta u^{m}+a u^{q} \int_{\Omega} u^{p}(y, t) d y, \quad x \in \Omega, t>0,
$$

subject to the homogeneous Dirichlet boundary and initial conditions

$$
\begin{aligned}
& u(x, t)=0, \quad x \in \partial \Omega, t>0, \\
& u(x, 0)=u_{0}(x), \quad x \in \Omega,
\end{aligned}
$$

where $0<m<1, p \geq 0, q>0, a>0$, and $\Omega \subset R^{N}(N \geq 1)$ is a bounded domain with smooth boundary and $u_{0} \in L^{\infty}(\Omega) \cap W_{0}^{1,2}(\Omega)$ is a nonnegative function. The symbols $\|\cdot\|_{p}(p \geq 1)$ and $|\Omega|$ denote the $L^{p}$-norm and the measure of $\Omega$, respectively.

Equation (1.1) describes the fast diffusion of concentration of some Newtonian fluids through porous media or the density of some biological species in many physical phenomena and biological species theories. It has been known that the nonlocal source term presents a more realistic model for population dynamics, see [1-3]. In the nonlinear diffusion theory, there exist obvious differences among the situations of slow $(m>1)$, fast $(0<m<1)$, and linear $(m=1)$ diffusions. For example, there is a finite speed propagation in the slow and linear diffusion situations, whereas an infinite speed propagation exists in the fast diffusion situation.

Recently, many scholars have been devoted to the study on blow-up and extinction properties of solutions for nonlinear diffusion equations with nonlocal terms, see [4-10]. Extinction of a function is a phenomenon for which there exists a finite time $T>0$ such that

\section{严 Springer}

○2013 Fang and Wang; licensee Springer. This is an Open Access article distributed under the terms of the Creative Commons Attribution License (http://creativecommons.org/licenses/by/2.0), which permits unrestricted use, distribution, and reproduction in any medium, provided the original work is properly cited. 
the solution is nontrivial on $(0, T)$ and then $u(x, t) \equiv 0$ for all $(x, t) \in \Omega \times[T,+\infty)$. In this case, $T$ is called an extinction time. It is also an important property of solutions to nonlinear parabolic equations which have been studied by many researchers. For example, Kalashnikov [11] studied the Cauchy problem of a semilinear parabolic equation with an absorption term

$$
u_{t}=\Delta u-\lambda u^{p}, \quad x \in R^{N}, t>0,
$$

and obtained extinctions as well as localization and finite propagation properties of the solutions. Evans and Knerr [12] investigated extinction behaviors of the solutions for the Cauchy problem of a semilinear parabolic equation with a fully nonlinear absorption term

$$
u_{t}(x, t)=\Delta u(x, t)-\beta(u(x, t)), \quad x \in R^{N}, t>0 .
$$

Ferrieira and Vazquez [13] studied extinction phenomena of the solutions for the Cauchy problem of a porous medium equation with an absorption term

$$
u_{t}=\left(u^{m}\right)_{x x}-u^{p}, \quad x \in R, t>0,
$$

by using the analysis of a self-similar solution. By constructing a suitable comparison function, $\mathrm{Li}$ and $\mathrm{Wu}$ [14] considered the problem of a porous medium equation with a local source term

$$
u_{t}=\Delta u^{m}+\lambda u^{p}, \quad x \in \Omega, t>0,
$$

subject to homogeneous Dirichlet boundary condition (1.2) and initial condition (1.3). They obtained some conditions for extinction and non-extinction of the solutions to the above equation and decay estimates. On extinctions of solutions to the $p$-Laplacian equation or the doubly degenerate equations, refer to $[15,16]$ and the references therein.

For equation (1.1) with $p=0$ and $N>2$, Han and Gao [8] showed that $q=m$ is a critical exponent for occurrence of extinction or non-extinction. Recently, Fang and Xu [9] considered equation (1.1) with $p=0$ and a linear absorption term, when the diffusion term was replaced with $p$-Laplacian operator in the whole dimensional space, and showed that the extinction of the weak solution is determined by competition of source and absorption terms. They also obtained the exponential decay estimates which depend on the initial data, coefficients, and domains. Thereafter, they obtained the same results for a class of nonlocal porous medium equations with strong absorption, see [10].

Motivated by the mentioned works above, we study extinction behaviors of the solutions for problem (1.1)-(1.3) in the whole dimensional space. The main tools we use are the super- and sub-solution and the energy methods to obtain some sufficient conditions for extinction of the solutions, and we give exponential decay estimates which depend on the initial data, coefficients, and domains. In fact, the energy method has a wide application, especially for the equations that do not satisfy the maximum principle (cf. [17]).

Our paper is organized as follows. In Section 2, we give preliminary knowledge including lemmas that are required in the proofs of our results. In Section 3, we obtain a critical value for extinction of the solutions to problem (1.1)-(1.3) by using the modified comparison principle and give the decay estimates for the extinctions of the solutions in Section 4. 


\section{Preliminaries and main results}

Due to the singularity of equation (1.1), problem (1.1)-(1.3) has no classical solutions in general, and hence it is reasonable to find a weak solution of the problem. To this end, we first give the following definition of a weak local solution.

Definition 1 A function $u \in L^{\infty}\left(Q_{T}\right)$ is called a super-solution of problem (1.1)-(1.3) in $Q_{T}$ if the following conditions hold:

(i) $u(x, 0) \geq u_{0}(x)$ in $\Omega$,

(ii) $u(x, t) \geq 0$ on $\partial \Omega \times(0, T)$,

(iii) for every $t \in(0, T)$ and every test function $\xi$,

$$
\begin{aligned}
\int_{\Omega} u(x, t) \xi(x, t) d x \geq & \int_{\Omega} u_{0}(x) \xi(x, 0) d x \\
& +\int_{0}^{t} \int_{\Omega}\left\{u \xi_{s}+u^{m} \Delta \xi+a u^{q} \int_{\Omega} u^{p}(y, t) d y \xi(x, s)\right\} d x d s,
\end{aligned}
$$

where $0 \leq \xi \in C\left(\overline{Q_{T}} \cap C^{1,2}\left(Q_{T}\right), \xi_{t}, \Delta \xi \in L^{2}\left(Q_{T}\right), \xi \geq 0, \xi_{\partial \Omega \times(0, T)}=0\right.$, and $Q_{T}=\Omega \times(0, T)$. A sub-solution can be similarly defined by replacing the inequality sign $\geq$ in the above conditions with $\leq$. A function is called a local solution of (1.1)-(1.3) if it is both super- and sub-solution for some $T$.

Let $\varphi(x)$ be the unique positive solution of the following linear elliptic problem:

$$
-\triangle \varphi(x)=1, \quad x \in \Omega ; \quad \varphi(x)=0, \quad x \in \partial \Omega .
$$

Throughout this paper, the constants $M$ and $\mu$ are defined as $M=\sup _{x \in \bar{\Omega}} \varphi(x)$ and $\mu=$ $\int_{\Omega} \varphi^{\frac{p}{m}}(x) d x$. The existence of local solutions can be obtained by utilizing the method of the standard regularization, and the regularities of the solutions can be derived by the argument similar to that in [18]. Since the regularization procedure is important to showing the uniqueness of the solution to problem (1.1)-(1.3) for some special cases, we sketch the outline below.

Consider the regularized problem

$$
\begin{aligned}
& u_{t}=\Delta u^{m}+a u^{q} \int_{\Omega} u^{p}(y, t) d y, \quad x \in \Omega, 0<t<T, \\
& u(x, t)=\frac{1}{k}, \quad x \in \partial \Omega, 0<t<T, \\
& u(x, 0)=u_{0}(x)+\frac{1}{k}, \quad x \in \Omega,
\end{aligned}
$$

where $T>0$ can be chosen sufficiently small so that there exists a solution $u_{k}$ of (2.2) on $Q_{T}$ for every $k \in N$ and $\left\|u_{k}\right\|_{\infty}$ is bounded for all $k$. Furthermore, $\frac{1}{l} \leq u_{l} \leq u_{k}$ for $k<l$, and a super-solution (sub-solution) comparison theory holds for (2.2) (see [19]).

Since the sequence $\left\{u_{k}\right\}$ is monotone and bounded, we may define $U(x, t) \equiv \lim _{k \rightarrow \infty} u_{k}(x$, $t$ ), and it is easy to see that $U(x, t)$ is a solution of (1.1)-(1.3). Furthermore, if $u$ is a solution 
of (1.1)-(1.3), we then have

$$
\begin{aligned}
\int_{\Omega}\left(u-u_{k}\right) \xi(x, t) d x= & \int_{0}^{t} \int_{\Omega}\left\{\left(u-u_{k}\right) \xi_{s}+\left(u^{m}-u_{k}^{m}\right) \triangle \xi\right. \\
& \left.+a \xi(x, s)\left(u^{q} \int_{\Omega} u^{p}(y, t) d y-u_{k}^{q} \int_{\Omega} u_{k}^{p}(y, t) d y\right)\right\} d x d s \\
& +\frac{1}{k} \int_{0}^{t} \int_{\Omega} \frac{\partial \xi}{\partial n} d S_{x} d s-\frac{1}{k} \int_{\Omega} \xi(x, 0) d x \\
\leq & \int_{0}^{t} \int_{\Omega}\left\{\left(u-u_{k}\right) \xi_{s}+\left(u^{m}-u_{k}^{m}\right) \Delta \xi\right. \\
& \left.+a \xi(x, s)\left(u^{q} \int_{\Omega} u^{p}(y, t) d y-u_{k}^{q} \int_{\Omega} u_{k}^{p}(y, t) d y\right)\right\} d x d s \\
= & \int_{0}^{t} \int_{\Omega}\left\{\left(u-u_{k}\right) \xi_{s}+\left(u^{m}-u_{k}^{m}\right) \triangle \xi+a \xi \int_{\Omega} u^{p}(y, t) d y\left(u^{q}-u_{k}^{q}\right)\right. \\
& \left.+a \xi(x, s) u_{k}^{q} \int_{\Omega}\left(u^{p}(y, t)-u_{k}^{p}(y, t) d y\right)\right\} d x d s .
\end{aligned}
$$

Here, we have used the fact $\frac{\partial \xi}{\partial n} \leq 0$ on $\partial \Omega$ to derive this inequality.

Define the functions $\Phi, F$, and $G$ as

$$
\begin{aligned}
& \Phi(x, t)=m \int_{0}^{1}\left(\theta u+(1-\theta) u_{k}\right)^{m-1} d \theta, \\
& F(x, t)=q \int_{0}^{1}\left(\theta u+(1-\theta) u_{k}\right)^{q-1} d \theta,
\end{aligned}
$$

and

$$
G(x, t)=p \int_{0}^{1}\left(\theta u+(1-\theta) u_{k}\right)^{p-1} d \theta,
$$

and let $\varphi_{u}=u^{m}$, we then have

$$
\begin{aligned}
\Phi & =\int_{0}^{t} \varphi_{u}\left(\theta u+(1-\theta) u_{k}\right) d \theta \geq \int_{0}^{\frac{1}{2}} \varphi_{u}\left(\frac{u_{k}}{2}\right) d \theta \\
& \geq \frac{1}{2} \min _{x \in \Omega, s \in[0, t] \max \left\{u+u_{k}(x, t) \in Q_{T}\right\} \geq p \geq \frac{1}{l}} \varphi_{u}(p) \equiv \eta,
\end{aligned}
$$

and we also have $F, G \in L^{\infty}$. We can choose sequences of smooth functions $\Phi_{n}, F_{n}$, and $G_{n}$ such that $\Phi_{n} \rightarrow \Phi, F_{n} \rightarrow F$, and $G_{n} \rightarrow G$ in $L^{2}\left(Q_{t_{1}}\right)$ and also find a constant $\gamma$ for which $\eta<\Phi_{n}<\gamma$ for all $n$.

Let $\xi \equiv \xi_{n}$ denote the solution of the problem

$$
\begin{aligned}
& \xi_{s}+\Phi_{n} \Delta \xi+a \xi M^{p}|\Omega| G_{n}+a M^{q} \mu F_{n}=0, \quad x \in \Omega, t \in(0, T), \\
& \xi(x, t)=\chi(x), \quad x \in \Omega,
\end{aligned}
$$

where $\chi$ is a smooth function and has compact support in $\Omega$ with $0 \leq \chi \leq 1$. From Lemma 4.2 in [19], we can find some constants $C_{1}$ and $C_{2}$ such that $0 \leq \xi \leq C_{1}$ and 
$\|\nabla \xi(\cdot, t)\|_{2},\|\triangle \xi\|_{2},\|\nabla \xi\|_{2} \leq C_{2}$. With this $\xi$, we have

$$
\begin{aligned}
& \int_{\Omega}\left(u-u_{k}\right)(x, t) \chi(x, t) d x \\
& \quad \leq \int_{0}^{t} \int_{\Omega}\left(u-u_{k}\right)\left(\Phi-\Phi_{n}\right) \Delta \xi+a \xi M^{p}|\Omega|\left(G-G_{n}\right)+a M^{q} \mu\left(F-F_{n}\right) d x d s .
\end{aligned}
$$

Since $u, u_{k} \in L^{\infty}\left(Q_{T}\right)$ for some constant $M$ and $u_{0} \leq v_{0}$, we deduce that

$$
\begin{aligned}
\int_{\Omega}\left(u-u_{k}\right)(x, t) \chi(x, t) \leq & K\left(\left\|\Phi_{n}-\Phi\right\|_{2}\|\Delta \xi\|_{2}+a M^{p}|\Omega|\left\|G-G_{n}\right\|_{2}\|\xi\|_{2}\right. \\
& \left.+a M^{q}|\Omega|\|\mu\| F-F_{n} \|_{2}\right)
\end{aligned}
$$

for some constant $K$. Letting $n \rightarrow \infty$, we obtain

$$
\int_{\Omega}\left(u-u_{k}\right)(x, t) \chi(x, t) d x \leq 0
$$

Choosing $\chi=\operatorname{sign}\left(u-u_{k}\right)^{+}$, we have

$$
\int_{\Omega}\left(u-u_{k}\right)^{+}(x, t) d x \leq 0
$$

This implies that $u \leq u_{k}$ on $Q_{t_{1}}$. Since $t_{1}<T$ is arbitrary, we have $u \leq u_{k} \leq U$ on $Q_{T}$.

To establish the uniqueness of solution to problem (1.1)-(1.3) for some special cases, it only remains to prove that the reverse inequality is also true. The desired result can be seen in the following proposition.

Proposition 1 If $p+q=m$ and $a \mu M^{\frac{q}{m}}<1$, where $\mu$ and $M$ are constants defined in (2.1), then the nonnegative solution of (1.1)-(1.3) is unique. Furthermore, if $v$ is a sub-solution of (1.1)-(1.3), we have $v \leq u$.

Proof We only need to show the uniqueness of solution.

Let $u$ be an arbitrary solution of (1.1)-(1.3), and let $u_{k}$ denote the solution of (2.2). We then have

$$
\begin{aligned}
\int_{\Omega}\left(u_{k}-u\right) \xi(x, t) d x \\
=\int_{0}^{t} \int_{\Omega}\left\{\left(u_{k}-u\right) \xi_{s}+\left(u_{k}^{m}-u^{m}\right) \triangle \xi\right. \\
\left.\quad+a \xi(x, s)\left(u_{k}^{q} \int_{\Omega} u_{k}^{p}(y, t) d y-u^{q} \int_{\Omega} u^{p}(y, t) d y\right)\right\} d x d s \\
\quad-\frac{1}{k} \int_{0}^{t} \int_{\Omega} \frac{\partial \xi}{\partial n} d S_{x} d s+\frac{1}{k} \int_{\Omega} \xi(x, 0) d x .
\end{aligned}
$$


Choosing the unique positive solution $\varphi(x)$ of $(2.1)$ as a testing function $\xi(x, t)$ and noticing that $p+q=m, a \mu M^{\frac{q}{m}}<1$, and $0 \leq u \leq u_{k} \leq M^{\prime}$, we get

$$
\begin{aligned}
\int_{\Omega}\left(u_{k}-u\right) \xi(x, t) d x= & \int_{0}^{t} \int_{\Omega}\left\{-\left(u_{k}-u\right)+a \mu M^{\frac{q}{m}}\left(M^{p+q}-0^{p+q}\right)\right\} d x d s \\
& -\frac{1}{k} \int_{0}^{t} \int_{\Omega} \frac{\partial \xi}{\partial n} d S_{x} d s+\frac{1}{k} \int_{\Omega} \xi(x, 0) d x \\
\leq & -\frac{1}{k} \int_{0}^{t} \int_{\Omega} \frac{\partial \xi}{\partial n} d S_{x} d s+\frac{1}{k} \int_{\Omega} \xi(x, 0) d x .
\end{aligned}
$$

Letting $k \rightarrow \infty$, we obtain

$$
\int_{\Omega}(U-u) \xi(x, t) d x \leq 0
$$

The above inequality together with the fact $U \geq u$ guarantees that $u \equiv U$.

The following comparison principle and lemmas will play a crucial role in what follows, but the proofs of them are simple, and so we omit them here (see $[19,20])$.

Proposition 2 (Comparison principle) Let $u$ and $v$ be nonnegative bounded super-and sub-solution of (1.1)-(1.3), respectively, with $u \geq \delta$ for some $\delta>0$. If $v(x, 0) \leq u(x, 0)$, then $v \leq u$ on $Q_{T}$.

Lemma 1 Suppose that $k$ and $\alpha$ are positive constants, with $k<1$. If $y(t)$ is a nonnegative absolutely continuous function on $[0, \infty)$ solving the problem

$$
\frac{d y}{d t}+\alpha y^{k} \leq 0, \quad t \geq 0 ; \quad y(0) \geq 0
$$

we then have the decay estimate

$$
\begin{aligned}
& y(t) \leq\left[y^{1-k}(0)-\alpha(1-k) t\right]^{\frac{1}{1-k}}, \quad t \in\left[0, T_{*}\right), \\
& y(t) \equiv 0, \quad t \in\left[T_{*},+\infty\right),
\end{aligned}
$$

where $T_{*}=\frac{y^{1-k}(0)}{\alpha(1-k)}$.

Lemma 2 [20] Suppose that $0<k<m \leq 1$ and $y(t)$ is a nonnegative function solving the problem

$$
\frac{d y}{d t}+\alpha y^{k} \leq \gamma y^{m}, \quad t \geq 0 ; \quad y(0)=y_{0}>0,
$$

where $\alpha$ and $\gamma$ are nonnegative constants. If $\gamma<\alpha y_{0}^{k-m}$, there exists $\eta>0$ such that $0 \leq$ $y(t) \leq y_{0} e^{-\eta t}$ for all $t \geq 0$.

\section{Extinction and non-extinction}

In this section, we construct suitable super- and sub-solution to determine whether there exist extinction phenomena for the solutions of problem (1.1)-(1.3). 
Theorem 1 If $p+q=m$ and $a \mu M^{\frac{q}{m}}<1$, then for any nonnegative initial data $u_{0}$, the unique solution $u(x, t)$ of (1.1)-(1.3) vanishes in finite time.

Proof We will prove this theorem by constructing a proper super-solution. Let $\varphi_{1}(x)$ be the unique positive solution of the following elliptic problem:

$$
-\Delta \varphi_{1}(x)=1, \quad x \in \Omega_{1} ; \quad \varphi_{1}(x)=0, \quad x \in \partial \Omega_{1} .
$$

Let $M_{1}=\sup _{x \in \bar{\Omega}_{1}} \varphi_{1}(x), \delta=\inf _{x \in \bar{\Omega}_{1}} \varphi_{1}(x)$, and let $\mu_{1}=\int_{\Omega_{1}} \varphi_{1}^{\frac{p}{m}}(x) d x$. We know from the comparison principle of elliptic problems that $\varphi(x)<\varphi_{1}(x)$ for $x \in \Omega$, and $\delta>0$ and $\mu<\mu_{1}$. Since $a \mu M^{\frac{q}{m}}<1$ and $\varphi_{1}(x)$ is continuous, we can find a domain $\Omega \subset \Omega_{1}$ such that $a \mu_{1} M_{1}^{\frac{q}{m}}<1$. Let $g(t)$ be the positive solution of the following problem:

$$
g^{\prime}(t)=-\frac{1-a \mu_{1} M_{1}^{\frac{q}{m}}}{M_{1}^{\frac{1}{m}}} g^{m}(t), \quad g(0)=A,
$$

where $A>0$ is a constant large enough so that $u_{0} \leq A \varphi_{1}^{\frac{1}{m}}(x)$ for all $x \in \bar{\Omega}$. Since $0<m<1$, the function $g(t)$ vanishes in a finite time $T_{0}>0$. Set $v(x, t)=g(t) \varphi_{1}^{\frac{1}{m}}(x)$, and then it can be easily seen that $v(x, t)$ also vanishes from the time $T_{0}$.

On the other hand, one can directly verify that $v(x, t)$ is a super-solution of (1.1)-(1.3) for any fixed $T$ such that $0<T<T_{0}$. There exist two positive constants $C_{1}$ and $C_{2}$ such that $C_{1} \leq v(x, t) \leq C_{2}$. By Proposition 2 , we know that $u(x, t) \leq v(x, t)$ for any $(x, t) \in \bar{\Omega} \times[0, T]$. Since $T<T_{0}$ is arbitrary, one can see that $u\left(x, T_{1}\right) \equiv 0$ for some $T_{1}<T_{0}$. Then it follows from Proposition 1 that $u(x, t) \equiv 0$ for all $t \geq T_{1}$, which implies that $u(x, t)$ vanishes from the time $T_{1}$.

Theorem 2 Suppose that $p+q>m$ and $a \leq \frac{\max \varphi \frac{p+q}{m}|\Omega|}{2 \delta^{p+q-m}}$. Then, for any nonnegative initial data $u_{0}$, the unique solution $u(x, t)$ of (1.1)-(1.3) vanishes in finite time.

Proof Let $v(x, t)=g(t) \varphi^{\frac{1}{m}}(x)$, where $\varphi(x)$ is the function solving (2.1). Then $v(x, t)$ is a super-solution of (1.1)-(1.3) if and only if the following conditions (3.2), (3.3), and (3.4) hold:

$$
\begin{aligned}
& g^{\prime} \varphi^{\frac{1}{m}}(x) \geq g^{m}(t) \Delta \varphi+a g^{p+q}(t) \varphi^{\frac{p}{m}} \int_{\Omega} \varphi^{\frac{q}{m}} d x, \quad x \in \Omega, 0<t<T, \\
& g(t) \varphi^{\frac{1}{m}}(x) \geq 0, \quad x \in \partial \Omega, 0<t<T, \\
& g(0) \varphi^{\frac{1}{m}}(x) \geq 0, \quad x \in \Omega .
\end{aligned}
$$

Let $g^{\prime}(t)=-\frac{g^{m}(t)}{2 H}$, where $\min _{x \in \Omega} \varphi(x)=1$ and $H=\max \varphi^{\frac{1}{m}}(x)$. One can see that the following condition is sufficient to guarantee (3.2):

$$
g^{m}(t) \geq-g^{\prime}(t) \varphi^{\frac{1}{m}}(x)+a g^{p+q}(t) \varphi^{\frac{p}{m}} \int_{\Omega} \varphi^{\frac{q}{m}} d x
$$

Since

$$
g^{\prime}(t) \varphi^{\frac{1}{m}}(x)=\frac{g^{m}(t)}{2 H} \varphi^{\frac{1}{m}} \leq \frac{1}{2} g^{m}(t)
$$


and $v(x, t) \geq \delta$, we may choose a constant $a \leq \frac{\max \varphi \frac{p+q}{m}|\Omega|}{2 \delta^{p+q-m}}$ such that

$$
a g^{p+q}(t) \varphi^{\frac{p}{m}} \int_{\Omega} \varphi^{\frac{q}{m}} d x \leq \frac{1}{2} g^{m}(t)
$$

Combining (3.6) with (3.7), it can be seen that (3.5) is true. Hence, if $u_{0} \leq g(0) \varphi(x)$, the function $v$ is a super-solution of (1.1)-(1.3). From the definition of $g(t)$, we obtain the integral equality

$$
\int_{g(t)}^{g_{0}} \frac{d s}{g^{m}}=\frac{t}{2 H}
$$

Define $\bar{T}=2 H \int_{0}^{g_{0}} \frac{1}{g^{m}} d s$. We then have $v>0$ for $0 \leq t<\bar{T}$ and $x \in \bar{\Omega}$, and $v(x, \bar{T})=0$ for $x \in \Omega$. By Proposition 2, we conclude that $u \leq v$ for $x \in \Omega$ and $0<t<\bar{T}$.

Theorem 3 Assume that $p+q<m$ or $p+q=m$ and $a \mu M^{\frac{q}{m}}>1$. Then, for any nonnegative initial data $u_{0}$, the maximal solution $U(x, t)$ of $(1.1)-(1.3)$ does not vanish in finite time.

Proof We will prove this theorem by constructing a suitable sub-solution. Set $v(x, t)=$ $g(t) \varphi^{\frac{1}{m}}$, where $\varphi(x)$ is the unique positive solution of (2.1). It can be easily verified that $v(x, t)$ is a sub-solution of (1.1)-(1.3) for $p+q<m$, if $g(t)$ solves the problem

$$
\begin{aligned}
& g^{\prime}(t)=M^{-\frac{1}{m}}\left\{-g^{m}(t)+a \mu M^{\frac{q}{m}} g^{p+q}\right\} ; \\
& g(0)=0, \quad g(t)>0 \quad(t>0) .
\end{aligned}
$$

If $p+q=m$ and $a \mu M^{\frac{q}{m}}>1$, choose $g(t)$ to be the solution of the problem

$$
\begin{aligned}
& g^{\prime}(t)=M^{-\frac{1}{m}}\left(a \mu M^{\frac{q}{m}}-1\right) g^{m}(t) ; \\
& g(0)=0, \quad g(t)>0 \quad(t>0) .
\end{aligned}
$$

Then $v(x, t)$ is also a sub-solution of (1.1)-(1.3). Hence, it follows from the sub-solution comparison principle that $U(x, t)>0$ in $\Omega \times(0, \infty)$.

Remark 1 It can be seen from Theorems 1-3 that when $p+q<m$ or $p+q=m$ and $a \mu M^{\frac{q}{m}}>1$, the maximal solution $U(x, t)$ is positive for all $t>0$, which means that the effect of the source term is, in some sense, strong and the diffusion term cannot dominate the source term. However, when $p+q>m$ and $a \leq \frac{\max \varphi^{\frac{p+q}{m}}|\Omega|}{2 \delta^{p+q-m}}$ or $p+q=m$ and $a \mu M^{\frac{q}{m}}<1$, the effect of the nonlocal source is a little weak, and the diffusion term may cause the nonnegative solution of (1.1)-(1.3) to vanish in finite time, provided that the initial data are sufficiently small.

\section{Decay estimates}

In Section 3, we have used the super- and sub-solution method to obtain some sufficient conditions on extinction for the solutions of problem (1.1)-(1.3), but we cannot obtain the decay estimates by the method. Therefore, we adopt other approximation techniques, i.e., the energy method and the differential inequality technique to obtain corresponding decay estimates which depend on the initial data, coefficients, and domains. 
Theorem 4 Suppose $0<m<1$ and $m=p+q$.

(1) When $N=1$ or 2 , if $|\Omega|<\left[\frac{4 m \gamma_{1}^{-2}(N, \Omega)}{a(m+1)^{2}}\right] \frac{2}{3-m}$ or $a<\frac{4 m \gamma_{1}^{-2}(N, \Omega)}{|\Omega|^{\frac{3-m}{2}}(m+1)^{2}}$, then for any nonnegative initial data $u_{0}$, the unique solution $u(x, t)$ of (1.1)-(1.3) vanishes in finite time, with the following decay estimates:

$$
\begin{aligned}
\|u(\cdot, t)\|_{2}^{2} \leq\left[\left\|u_{0}\right\|_{2}^{1-m}-C_{1}\left(1-\frac{m+1}{2}\right) t\right]^{\frac{1}{1-\frac{m+1}{2}}}, \quad t \in\left[0, T_{1}\right) ; \\
\|u(\cdot, t)\|_{2}^{2} \equiv 0, \quad t \in\left[T_{1},+\infty\right),
\end{aligned}
$$

where $\gamma_{1}>0$ is an embedding constant, and $C_{1}$ and $T_{1}$ will be determined later.

(2) When $N>2$,

(a) if $\frac{N-2}{N+2} \leq m<1$, and $|\Omega|<\left(\frac{\gamma_{2}^{-2}}{a}\right)^{\frac{1}{1+\frac{2}{N}}}$ or a $<\frac{\gamma_{2}^{-2}}{|\Omega|^{1+2}}$, then for any nonnegative initial data $u_{0}$, the unique solution $u(x, t)$ of (1.1)-(1.3) vanishes in finite time, with the following decay estimates:

$$
\begin{aligned}
\|u(\cdot, t)\|_{1+m}^{1+m} & \leq\left[\left\|u_{0}\right\|_{1+m}^{1-m}-C_{2}\left(1-\frac{2 m}{1+m}\right) t\right]^{\frac{1}{1-\frac{2 m}{1+m}}}, \quad t \in\left[0, T_{2}\right) ; \\
\|u(\cdot, t)\|_{1+m}^{1+m} & \equiv 0, \quad t \in\left[T_{2},+\infty\right),
\end{aligned}
$$

where $\gamma_{2}>0$ is an embedding constant, and $C_{2}$ and $T_{2}$ will be determined later;

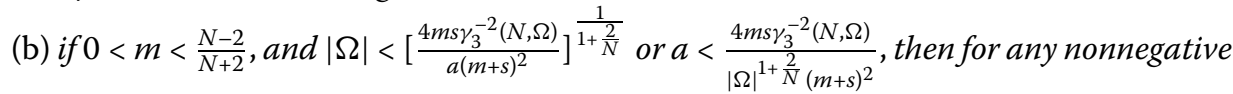
initial data $u_{0}$, the unique solution $u(x, t)$ of (1.1)-(1.3) vanishes in finite time, with the following decay estimates:

$$
\begin{aligned}
& \|u(\cdot, t)\|_{1+s}^{1+s} \leq\left[\left\|u_{0}\right\|_{1+s}^{1-m}-C_{3}\left(1-\frac{m+s}{1+s}\right) t\right]^{\frac{1}{1-\frac{m+s}{1+s}}}, \quad t \in\left[0, T_{3}\right) ; \\
& \|u(\cdot, t)\|_{1+s}^{1+s} \equiv 0, \quad t \in\left[T_{3},+\infty\right),
\end{aligned}
$$

where $s=\frac{N}{2}(1-m)-1$ and $\gamma_{3}>0$ is an embedding constant, and $C_{3}$ and $T_{3}$ will be determined later.

Proof We first consider the case $N=1$ or 2 . Multiplying both sides of equation (1.1) by $u^{s}$ $(s \geq 0)$ and integrating the result over $\Omega$, we obtain

$$
\frac{1}{s+1} \frac{d}{d t} \int_{\Omega} u^{s+1} d x+\frac{4 m s}{(m+s)^{2}} \int_{\Omega}\left|\nabla u^{\frac{m+s}{2}}\right|^{2} d x=a \int_{\Omega} u^{p} d x \int_{\Omega} u^{q+s} d x .
$$

By Hölder's inequality, we get the inequality

$$
\int_{\Omega} u^{p} d x \int_{\Omega} u^{q+s} d x \leq\|\Omega\|^{\frac{2 s_{1}-p-q-s}{s_{1}}}\|u\|_{s_{1}}^{p+q+s}
$$

where $s_{1} \geq 1$ will be determined later. Choosing $s_{1}=s+1 \geq 1$, we get the inequality

$$
\frac{1}{s+1} \frac{d}{d t} \int_{\Omega} u^{s+1} d x+\frac{4 m s}{(m+s)^{2}} \int_{\Omega}\left|\nabla u^{\frac{m+s}{2}}\right|^{2} d x \leq a|\Omega|^{\frac{2+s-m}{s+1}}\|u\|_{s+1}^{m+s} .
$$


By using the Sobolev embedding inequality, one can show that there exists an embedding constant $\gamma_{1}(N, \Omega)$ such that

$$
\left\|u^{\frac{m+s}{2}}\right\|_{s_{2}} \leq \gamma_{1}^{2}(N, \Omega)\left\|\nabla u^{\frac{m+s}{2}}\right\|_{2}
$$

where $s_{2} \geq 2$ will be determined later, i.e.,

$$
\|u\|_{\frac{(m+s) s_{2}}{2}}^{s+m} \leq \gamma_{1}^{2}(N, \Omega)\left\|\nabla u^{\frac{m+s}{2}}\right\|_{2}
$$

Let $C_{1}=\frac{4 m \gamma_{1}^{-2}(N, \Omega)}{(m+1)^{2}}-a|\Omega|^{\frac{3-m}{2}}, s_{2}=\frac{4}{1+m}$, and let $s=1$. Then $s_{2} \geq 2$, since $0<m<1$. From the above inequality with the constant $s_{2}$ and $s$, we obtain the inequality $\frac{d}{d t}\|u\|_{2}^{2}+C_{1}\|u\|_{2}^{m+1} \leq$ 0 , if $C_{1}>0$, i.e., $|\Omega|<\left[\frac{4 m \gamma_{1}^{-2}(N, \Omega)}{a(m+1)^{2}}\right]^{\frac{2}{3-m}}$. By Lemma 1 , we then obtain its decay estimates, with $T_{1}=\frac{\left\|u_{0}\right\|_{2}^{1-m}}{C_{1}\left(1-\frac{m+1}{2}\right)}$.

Secondly, we consider the case $N>2$.

(a) For $m$ such that $\frac{N-2}{N+2} \leq m<1$, multiplying both sides of equation of (1.1) by $u^{m}$ and integrating the result over $\Omega$, we obtain

$$
\frac{1}{m+1} \frac{d}{d t} \int_{\Omega} u^{m+1} d x+\int_{\Omega}\left|\nabla u^{m}\right|^{2} d x=a \int_{\Omega} u^{p} d x \int_{\Omega} u^{q+m} d x
$$

By the Sobolev embedding and Hölder's inequalities, one can show that

$$
\frac{1}{m+1} \frac{d}{d t}\|u\|_{1+m}^{(1+m)}+\gamma_{2}^{-2}|\Omega|^{\frac{N-2}{N}-\frac{2 m}{1+m}}\|u\|_{1+m}^{2 m} \leq a|\Omega|^{\frac{2}{1+m}}\|u\|_{1+m}^{2 m}
$$

and hence we get

$$
\frac{d}{d t}\|u\|_{1+m}^{1+m}+C_{2}\|u\|_{1+m}^{2 m} \leq 0
$$

provided $C_{2}=(m+1)\left(\gamma_{2}^{-2}|\Omega|^{\frac{N-2}{N}-\frac{2 m}{1+m}}-a|\Omega| \frac{2}{1+m}\right)>0$, i.e., $|\Omega|<\left(\frac{\gamma_{2}^{-2}}{a}\right)^{\frac{1}{1+\frac{2}{N}}}$. By Lemma 1, we then obtain its decay estimates, with $T_{2}=\frac{\left\|u_{0}\right\|_{1+m}^{1-m}}{C_{2}\left(1-\frac{2 m}{1+m}\right)}$.

(b) For $m$ such that $0<m<\frac{N-2}{N+2}$, multiplying both sides of equation (1.1) by $u^{s}\left(s=\frac{N}{2}(1-\right.$ $m)-1$ ) and integrating the result over $\Omega$, we obtain

$$
\frac{1}{s+1} \frac{d}{d t} \int_{\Omega} u^{s+1} d x+\frac{4 m s}{(m+s)^{2}} \int_{\Omega}\left|\nabla u^{\frac{m+s}{2}}\right|^{2} d x=a \int_{\Omega} u^{p} d x \int_{\Omega} u^{q+s} d x
$$

By the Sobolev embedding inequality and a suitable $s$, one can show that

$$
\|u\|_{s+1}^{\frac{s+m}{2}}=\left(\int_{\Omega} u^{\frac{s+m}{2}-\frac{2 N}{N-2}}\right) \leq \gamma_{3}\left\|\nabla u^{\frac{s+m}{2}}\right\|_{2} .
$$

Using Hölder's inequality, we have

$$
\int_{\Omega} u^{p} d x \int_{\Omega} u^{q+s} d x \leq\|\Omega\|^{2-\frac{m+s}{s+1}}\|u\|_{s+1}^{m+s} .
$$


From inequalities (4.5) and (4.6), we get the inequality

$$
\frac{1}{s+1} \frac{d}{d t}\|u\|_{1+s}^{(1+s)}+\frac{4 m s}{(m+s)^{2}} \gamma_{3}^{-2}\|u\|_{1+s}^{s+m} \leq a|\Omega|^{2-\frac{m+s}{1+s}}\|u\|_{1+s}^{s+m} .
$$

Furthermore, we can have $\frac{d}{d t}\|u\|_{1+s}^{1+s}+C_{3}\|u\|_{1+s}^{s+m} \leq 0$, provided $C_{3}=(s+1)\left(\gamma_{3}^{-2} \frac{4 m s}{(m+s)^{2}}-\right.$ $\left.a|\Omega|^{2-\frac{m+s}{1+s}}\right)>0$, i.e., $|\Omega|<\left(\frac{4 m s \gamma_{3}^{-2}}{a(m+s)^{2}}\right)^{\frac{1}{1+\frac{2}{N}}}$. By Lemma 1 , we then obtain its decay estimates, with $T_{3}=\frac{\left\|u_{0}\right\|_{1+s}^{1-m}}{C_{3} \frac{1-m}{1+s}}$.

Theorem 5 Suppose that $0<m<1$ and $p+q>m$.

(1) When $N=1$ or 2 , if

$$
|\Omega|<\left[\frac{8 m \gamma_{4}^{-2}(N, \Omega)}{a\left(\left\|u_{0}\right\|_{2}^{2} e^{-\alpha_{1} T_{4}}\right)^{p+q-m}(m+1)^{2}}\right]^{\frac{2}{3-p-q}}
$$

or

$$
a<\frac{8 m \gamma_{4}^{-2}(N, \Omega)}{|\Omega|^{\frac{3-p-q}{2}}\left(\left\|u_{0}\right\|_{2}^{2} e^{-\alpha_{1} T_{4}}\right)^{p+q-m}(m+1)^{2}}
$$

and $u_{0}$ small enough, the unique solution $u(x, t)$ of (1.1)-(1.3) vanishes in finite time, with the following decay estimates:

$$
\begin{aligned}
\|u(\cdot, t)\|_{2}^{2} & \leq\left[\left\|u_{0}\right\|_{2}^{2} e^{-\alpha_{1} t}\right], \quad t \in\left[0, T_{4}\right) ; \\
\|u(\cdot, t)\|_{2}^{2} & \leq\left[\left\|u_{0}\right\|_{2}^{1-m}-C_{4}\left(1-\frac{m+1}{2}\right) t\right]^{\frac{1}{1-\frac{m+1}{2}}}, \quad t \in\left[T_{4}, T_{5}\right) ; \\
\|u(\cdot, t)\|_{2}^{2} & \equiv 0, \quad t \in\left[T_{5},+\infty\right),
\end{aligned}
$$

where $\gamma_{4}>0$ is an embedding constant, $\alpha_{1}$ is a suitable constant, and $C_{4}, T_{4}, T_{5}$ will be determined later.

(2) When $N>2$,

(a) for $m$ such that $\frac{N-2}{N+2} \leq m<1$, if

$$
|\Omega|<\left(\frac{\gamma_{5}^{-2}}{\left(a\left\|u_{0}\right\|_{1+m}^{1+m} e^{-\alpha_{2}} t\right)^{\frac{p+q-m}{1+m}}}\right)^{\frac{N+2}{N}+\frac{m-p-q}{1+m}}
$$

or

$$
a<\frac{\gamma_{5}^{-2}}{|\Omega|^{\frac{N+2}{N}+\frac{m-p-q}{1+m}}\left(\left\|u_{0}\right\|_{1+m}^{1+m} e^{-\alpha_{2}} t\right)^{\frac{p+q-m}{1+m}}}
$$

and $u_{0}$ small enough, the unique solution $u(x, t)$ of (1.1)-(1.3) vanishes in finite time, with the following decay estimates:

$$
\begin{aligned}
\|u(\cdot, t)\|_{1+m}^{1+m} & \leq\left[\left\|u_{0}\right\|_{1+m}^{1+m} e^{-\alpha_{2} t}\right], \quad t \in\left[0, T_{6}\right) \\
\|u(\cdot, t)\|_{1+m}^{1+m} & \leq\left[\left\|u_{0}\right\|_{1+m}^{1-m}-C_{5}\left(1-\frac{2 m}{1+m}\right) t\right]^{\frac{1}{1-\frac{2 m}{1+m}}}, \quad t \in\left[T_{6}, T_{7}\right)
\end{aligned}
$$




$$
\|u(\cdot, t)\|_{1+m}^{1+m} \equiv 0, \quad t \in\left[T_{7},+\infty\right)
$$

where $\gamma_{5}>0$ is an embedding constant, $\alpha_{2}$ is a suitable constant, and $C_{5}, T_{6}, T_{7}$ will be determined later;

(b) for $m$ such that $0<m<\frac{N-2}{N+2}$, if

$$
|\Omega|<\left(\frac{4 m s \gamma_{6}^{-2}}{a(m+s)^{2}\left(\left\|u_{0}\right\|_{1+s}^{1+s} e^{-\alpha_{3}} t\right)^{\frac{p+q-s}{1+s}}}\right)^{\frac{1}{2-\frac{m+p+q}{1+m}}}
$$

or

$$
a<\frac{4 m s \gamma_{6}^{-2}}{|\Omega|^{2-\frac{m+p+q}{1+m}}(m+s)^{2}\left(\left\|u_{0}\right\|_{1+s}^{1+s} e^{-\alpha_{3}} t\right)^{\frac{p+q-s}{1+s}}}
$$

and $u_{0}$ small enough, the unique solution $u(x, t)$ of (1.1)-(1.3) vanishes in finite time, with the following decay estimates:

$$
\begin{aligned}
& \|u(\cdot, t)\|_{1+s}^{1+s} \leq\left[\left\|u_{0}\right\|_{1+s}^{1+s} e^{-\alpha_{3} t}\right], \quad t \in\left[0, T_{8}\right) \\
& \|u(\cdot, t)\|_{1+s}^{1+s} \leq\left[\left\|u_{0}\right\|_{1+s}^{1-m}-C_{6}\left(1-\frac{s+m}{1+s}\right) t\right]^{\frac{1}{1-\frac{s+m}{1+s}}, \quad t \in\left[T_{8}, T_{9}\right)} \\
& \|u(\cdot, t)\|_{1+s}^{1+s} \equiv 0, \quad t \in\left[T_{9},+\infty\right),
\end{aligned}
$$

where $\gamma_{6}>0$ is an embedding constant, $\alpha_{3}$ is a suitable constant, and $C_{6}, T_{8}, T_{9}$ will be determined later.

Proof Multiplying both sides of equation (1.1) by $u^{s}(s \geq 0)$ and integrating the result over $\Omega$, we obtain

$$
\frac{1}{s+1} \frac{d}{d t} \int_{\Omega} u^{s+1} d x+\frac{4 m s}{(m+s)^{2}} \int_{\Omega}\left|\nabla u^{\frac{m+s}{2}}\right|^{2} d x=a \int_{\Omega} u^{p} d x \int_{\Omega} u^{q+s} d x
$$

We first consider the case $p+q \leq 1$.

(1) When $N=1$ or 2 , setting $s=1$ in (4.7), we get

$$
\frac{1}{2} \frac{d}{d t} \int_{\Omega} u^{2} d x+\frac{4 m}{(m+1)^{2}} \int_{\Omega}\left|\nabla u^{\frac{m+1}{2}}\right|^{2} d x=a \int_{\Omega} u^{p} d x \int_{\Omega} u^{q+1} d x
$$

Using Hölder's inequality and the Sobolev embedding inequality, one can show that

$$
\frac{1}{2} \frac{d}{d t}\|u\|_{2}^{2}+\frac{8 m \gamma_{4}^{-2}}{(m+1)^{2}}\|u\|_{2}^{m+1} \leq a|\Omega|^{\frac{3-p-q}{2}}\|u\|_{2}^{p+q+1}
$$

By Lemma 2, one can see that there exists a constant $\alpha_{1}>0$ such that $0 \leq\|u(\cdot, t)\|_{2}^{2} \leq$ $\left\|u_{0}\right\|_{2}^{2} e^{-\alpha_{1} t}$, provided $\left\|u_{0}\right\|_{2}^{2} \leq\left[\frac{8 m \gamma_{4}^{-2}}{a(m+1)^{2}|\Omega|^{\frac{2}{3-p-q}}}\right]^{\frac{1}{p+q-m}}$. Hence, there exists a constant $T_{4}>0$ such that

$$
\frac{8 m \gamma_{4}^{-2}}{(m+1)^{2}}-a|\Omega|^{\frac{3-p-q}{2}}\|u\|_{2}^{p+q-m} \geq \frac{8 m \gamma_{4}^{-2}}{(m+1)^{2}}-a|\Omega|^{\frac{3-p-q}{2}}\left(\left\|u_{0}\right\|_{2}^{2} e^{-\alpha_{1} T_{4}}\right)^{p+q-m}=C_{4}>0
$$


for all $t \in\left[T_{4},+\infty\right)$, and so $\frac{d}{d t}\|u\|_{2}^{2}+C_{4}\|u\|_{2}^{m+1} \leq 0$. By Lemma 1 we can obtain its decay estimate, with $T_{5}=\frac{\left\|u_{0}\right\|_{2}^{1-m}}{C_{4}\left(1-\frac{m+1}{2}\right)}+T_{4}$.

(2) When $N>2$,

(a) for $m$ such that $\frac{N-2}{N+2}<m<1$, we choose $s=m$ in (4.7). We then have the inequality

$$
\frac{1}{m+1} \frac{d}{d t}\|u\|_{m+1}^{m+1}+\gamma_{5}^{-2}|\Omega|^{\frac{N-2}{N}-\frac{2 m}{1+m}}\|u\|_{s+1}^{m+s} \leq a|\Omega|^{2-\frac{p+q+m}{m+1}}\|u\|_{m+1}^{p+q+m}
$$

by the Sobolev embedding and Hölder's inequalities. By Lemma 2, one can see that there exists a constant $\alpha_{2}>0$ such that $0 \leq\|u(\cdot, t)\|_{m+1}^{m+1} \leq\left\|u_{0}\right\|_{m+1}^{m+1} e^{-\alpha_{2} t}$, provided $\left\|u_{0}\right\|_{m+1}^{m+1} \leq$ $\left[\frac{(m+1) \gamma_{5}^{-2}}{a|\Omega|^{1+2} N \frac{m-p-q}{m+1}}\right]^{\frac{1}{p+q-m}}$. Then there exists a constant $T_{6}>0$ such that

$$
\begin{aligned}
& (1+m)\left(\gamma_{5}^{-2}|\Omega|^{\frac{N-2}{N}-\frac{2 m}{1+m}}-a|\Omega|^{2-\frac{m+p+q}{1+m}}\|u\|_{1+m}^{p+q-m}\right) \\
& \quad \leq(1+m)\left(\gamma_{5}^{-2}|\Omega|^{\frac{N-2}{N}-\frac{2 m}{1+m}}\right)-a|\Omega|^{2-\frac{m+p+q}{1+m}}\left(\left\|u_{0}\right\|_{m+1}^{m+1} e^{-\alpha_{2} T_{6}}\right)^{\frac{p+q-m}{1+m}} \equiv C_{5} \quad(>0)
\end{aligned}
$$

for all $t \in\left[T_{6},+\infty\right)$.

Hence, we get $\frac{d}{d t}\|u\|_{m+1}^{m+1}+C_{5}\|u\|_{1+m}^{2 m} \leq 0$. By Lemma 1 we can obtain its decay estimate, with $T_{7}=\frac{\left\|u_{0}\right\|_{1-m}^{m+1}}{C_{6}\left(1-\frac{2 m}{m+1}\right)}+T_{6}$;

(b) for $m$ such that $0<m<\frac{N-2}{N+2}$, we choose $s=\frac{N}{2}(1-m)-1$ in (4.4). From the Sobolev embedding inequality, we get

$$
\|u\|_{s}^{\frac{m+s}{2}}=\left\|u^{\frac{m+s}{2}}\right\|_{\frac{2 N}{N-2}} \leq \gamma_{6}\left\|\nabla u^{\frac{m+s}{2}}\right\|_{2}
$$

By Hölder's inequality, we obtain the inequality

$$
\frac{1}{s+1} \frac{d}{d t}\|u\|_{s+1}^{s+1}+\frac{\gamma_{6}^{-2} 4 m s}{(m+s)^{2}}\|u\|_{s+1}^{m+s} \leq a|\Omega|^{2-\frac{p+q+s}{s+1}}\|u\|_{s+1}^{p+q+s} .
$$

One can see that there exists a constant $\alpha_{3}>0$ such that $0 \leq\|u(\cdot, t)\|_{s+1}^{s+1} \leq\left\|u_{0}\right\|_{s+1}^{s+1} e^{-\alpha_{3} t}$ by Lemma 2, provided $\left\|u_{0}\right\|_{s+1}^{s+1} \leq\left[\frac{4 m s \gamma_{6}^{-2}}{a(m+1)^{2}|\Omega|^{2-\frac{s+p+q}{s+1}}}\right]^{\frac{1}{p+q-s}}$. Then there exists a constant $T_{8}>0$ such that

$$
\begin{aligned}
(1 & +m)\left(\frac{4 s m \gamma_{6}^{-2}}{(m+s)^{2}}-a|\Omega|^{2-\frac{s+p+q}{1+s}}\|u\|_{1+s}^{p+q-s}\right) \\
& \leq(1+m) \frac{4 s m \gamma_{6}^{-2}}{(m+s)^{2}}-a|\Omega|^{2-\frac{s+p+q}{1+s}}\left(\left\|u_{0}\right\|_{s+1}^{s+1} e^{-\alpha_{3} T_{8}}\right)^{\frac{p+q-s}{1+s}} \equiv C_{6} \quad(>0)
\end{aligned}
$$

for all $t \in\left[T_{8},+\infty\right)$.

Hence, $\frac{d}{d t}\|u\|_{s+1}^{s+1}+C_{6}\|u\|_{s+1}^{m+s} \leq 0$. By Lemma 1 we can obtain its decay estimate, with $T_{9}=\frac{\left\|u_{0}\right\|_{1-m}^{s+1}}{C_{6}\left(1-\frac{m+s}{s+1}\right)}+T_{8}$.

Secondly, we consider the case $p+q>1$.

It can be easily verified that $k \varphi_{1}(x)$ is a super-solution of (1.1)-(1.3) for sufficiently small $k>0$, where $\varphi_{1}(x)$ is the unique positive solution of (2.1). We then have

$$
u(x, t) \leq k \varphi_{1}^{\frac{1}{m}}(x) \leq k M_{1}^{\frac{1}{m}}, \quad t>0
$$

by Proposition 2, if $u_{0} \leq k \varphi_{1}^{\frac{1}{m}}(x)$ in $\Omega$. 
The above inequality and (4.7) yield the inequality

$$
\frac{1}{s+1} \frac{d}{d t} \int_{\Omega} u^{s+1} d x+\frac{4 m s}{(m+s)^{2}} \int_{\Omega}\left|\nabla u^{\frac{m+s}{2}}\right|^{2} d x \leq a k^{p+q-1} M_{1}^{\frac{p+q-1}{m}}|\Omega| \int_{\Omega} u^{s+1} d x
$$

by applying Hölder's inequality to the right-hand side of (4.7).

For $m$ such that $\frac{N-2}{N+2}<m<1$, we choose $s=m$ in (4.11). It follows from the Sobolev embedding and Hölder's inequalities that

$$
\frac{1}{m+1} \frac{d}{d t}\|u\|_{m+1}^{m+1}+\gamma_{7}^{-2}|\Omega|^{\frac{N-2}{N}-\frac{2 m}{1+m}}\|u\|_{m+1}^{2 m} \leq a k^{p+q-1} M_{1}^{\frac{p+q-1}{m}}\|u\|_{m+1}^{1+m}
$$

By Lemma 2, there exists a constant $\alpha_{4}>0$ such that $0 \leq\|u(\cdot, t)\|_{m+1}^{m+1} \leq\left\|u_{0}\right\|_{m+1}^{m+1} e^{-\alpha_{4} t}$, provided $\left\|u_{0}\right\|_{m+1}^{m+1} \leq\left[\frac{|\Omega|^{\frac{N-2}{2}-\frac{2 m}{1+m} \gamma_{7}^{-2}}}{a k^{p+q-1} M_{1}^{\frac{p+q-1}{m}}}\right]^{\frac{1}{1-m}}$. Hence, one can find a constant $T_{10}>0$ such that

$$
\begin{aligned}
& (1+m)\left(\gamma_{7}^{-2}|\Omega|^{\frac{N-2}{N}-\frac{2 m}{1+m}}-a k^{p+q-1} M_{1}^{\frac{p+q-1}{m}}\|u\|_{1-m}^{1+m}\right) \\
& \quad \leq(1+m)\left(\gamma_{7}^{-2}|\Omega|^{\frac{N-2}{N}-\frac{2 m}{1+m}}\right)-a k^{p+q-1} M_{1}^{\frac{p+q-1}{m}}\left(\left\|u_{0}\right\|_{m+1}^{m+1} e^{-\alpha_{4} T_{10}}\right)^{\frac{1-m}{1+m}} \equiv C_{7} \quad(>0)
\end{aligned}
$$

for all $t \in\left[T_{10},+\infty\right)$, from which we get

$$
\frac{d}{d t}\|u\|_{m+1}^{m+1}+C_{7}\|u\|_{1+m}^{2 m} \leq 0
$$

By Lemma 1, we then obtain its decay estimates, with $T_{11}=\frac{\left\|u_{0}\right\|_{1-m}^{m+1}}{C_{7}\left(1-\frac{2 m}{m+1}\right)}+T_{10}$, as follows:

$$
\begin{aligned}
& \|u(\cdot, t)\|_{1+m}^{1+m} \leq\left[\left\|u_{0}\right\|_{1+m}^{1+m} e^{-\alpha_{4} t}\right], \quad t \in\left[0, T_{10}\right) ; \\
& \|u(\cdot, t)\|_{1+m}^{1+m} \leq\left[\left\|u_{0}\right\|_{1+m}^{1-m}-C_{7}\left(1-\frac{2 m}{1+m}\right) t\right]^{\frac{1}{1-\frac{2 m}{1+m}}}, \quad t \in\left[T_{10}, T_{11}\right) \\
& \|u(\cdot, t)\|_{1+m}^{1+m} \equiv 0, \quad t \in\left[T_{11},+\infty\right) .
\end{aligned}
$$

For $m$ such that $0<m<\frac{N-2}{N}$, we choose $s=\frac{N}{2}(1-m)>1$ in (4.7), and then we can obtain the extinction results by using a similar argument as the one used for the case $p+q \leq 1$, and so the details are omitted.

\section{Competing interests}

The authors declare that they have no competing interests.

\section{Authors' contributions}

All authors contributed equally to the manuscript and read and approved the final manuscript.

\section{Acknowledgements}

This work is supported by the Natural Science Foundation of Shandong Province of China (ZR2012AM018) and the Fundamental Research Funds for the Central Universities (No. 201362032). The authors would like to deeply thank all the reviewers for their insightful and constructive comments. 


\section{References}

1. Bebernes, J, Eberly, D: Mathematical Problems from Combustion Theory. Springer, New York (1989)

2. Pao, CV: Nonlinear Parabolic and Elliptic Equations. Plenum Press, New York (1992)

3. Furter, J, Grinfield, M: Local vs. non-local interactions in populations dynamics. J. Math. Biol. 27, 65-80 (1989)

4. Souplet, P: Blow-up in nonlocal reaction-diffusion equations. SIAM J. Math. Anal. 29, 1301-1334 (1998)

5. Souplet, P: Uniform blow-up profiles and boundary behavior for diffusion equations with nonlocal nonlinear source. J. Differ. Equ. 153, 374-406 (1999)

6. Li, FC, Xie, CH: Global and blow-up solutions to $p$-Laplacian equation with nonlocal source. Comput. Math. Appl. 46, 1525-1533 (2003)

7. Liu, QL, Li, YX, Gao, HJ: Uniform blow-up rate for diffusion equations with nonlocal nonlinear source. Nonlinear Anal. 67, 1947-1957 (2007)

8. Han, YZ, Gao, WJ: Extinction for a fast diffusion equation with a nonlinear nonlocal source. Arch. Math. 97, 353-363 (2011)

9. Fang, ZB, Xu, XH: Extinction behavior of solutions for the $p$-Laplacian equations with nonlocal sources. Nonlinear Anal., Real World Appl. 13, 1780-1789 (2012)

10. $\mathrm{Xu}, \mathrm{XH}$, Fang, ZB, Yi, SC: Extinction and decay estimates of solutions for porous medium equation with nonlocal source and strong absorption. Bound. Value Probl. 2013, Article ID 24 (2013). doi:10.1186/1687-2770-2013-24

11. Kalashnikov, AS: The propagation of disturbances in problem of nonlinear heat conduction with absorption. USSR Comput. Math. Math. Phys. 14, 70-85 (1974)

12. Evans, LC, Knerr, BF: Instantaneous shrinking of the support of nonnegative solutions to certain nonlinear parabolic equations and variational inequalities. III. J. Math. 23, 153-166 (1979)

13. Ferreira, $R$, Vazquez, JL: Extinction behavior for fast diffusion equations with absorption. Nonlinear Anal. TMA 43, 353-376 (2001)

14. Li, YX, Wu, JC: Extinction for fast diffusion equations with nonlinear sources. Electron. J. Differ. Equ. 23, 1-7 (2005)

15. Tian, Y, Mu, CL: Extinction and non-extinction for a $p$-Laplacian equation with nonlinear source. Nonlinear Anal. TMA 69, 2422-2431 (2008)

16. Fang, ZB, Li, G: Extinction and decay estimates of solutions for a class of doubly degenerate equations. Appl. Math. Lett. 25, 1795-1802 (2012)

17. Antontsev, SN, Diaz, JI, Shmarev, S, Kassab, AJ: Energy Methods for Free Boundary Problems: Applications to Nonlinear PDEs and Fluid Mechanics. Progress in Nonlinear Differential Equations and Their Applications. Applied Mechanics Reviews. Birkhäuser, Auflage (2002)

18. Sacks, PE: Continuity of solutions of a singular parabolic equation. Nonlinear Anal. 7, 387-409 (1983)

19. Anderson, JR: Local existence and uniqueness of solutions of degenerate parabolic equation. Commun. Partial Differ. Equ. 16, 105-143 (1991)

20. Chen, SL: The extinction behavior of solution for a reaction-diffusion equation. J. Math. Res. Expo. 18, $583-586$ (1998)

10.1186/1687-2770-2013-266

Cite this article as: Fang and Wang: Extinction properties of solutions for a fast diffusion equation with nonlocal source. Boundary Value Problems 2013, 2013:266

\section{Submit your manuscript to a SpringerOpen ${ }^{\circ}$ journal and benefit from:}

- Convenient online submission

- Rigorous peer review

- Immediate publication on acceptance

Open access: articles freely available online

- High visibility within the field

- Retaining the copyright to your article 